\title{
ACTITUDES DE UN GRUPO DE BACHILLERES ASISTENCIALES EN SERVICIO DEL HOSPITAL UNIVERSITARIO “ANGELO LARRALDE” HACIA LA CARRERA DE ENFERMERÍA
}

El propósito de la presente investigación fue estudiar las actitudes de un grupo de bachilleres asistenciales en servicio hacia la carrera de Enfermería. El objetivo central fue determinar las actitudes en cuanto a: status social, económico, vocación y desarrollo científico en Enfermería. La población estuvo conformada por ciento treinta y ocho (138) bachilleres asistenciales del Hospital Universitario "Angel Larralde" en Valencia, Venezuela. La muestra de hizo al azar simple y representa el $30 \%$ del universo. El diseño utilizado fue el descriptivo. El instrumento es inédito y se seleccionó la escala de Lickert. Las técnicas estadísticas utilizadas fue el análisis de proporciones. Los hallazgos indican actitudes favorables en cuanto a status social, vocación y desarrollo científico y desfavorable en cuanto a status económico de acuerdo a los porcentajes significativos obtenidos. Esta investigación se realizó como requisito de pregrado en la Escuela de la Universidad de Carabobo, en junio de 1990.

\section{Problema}

Actitudes de un grupo de bachilleres asistenciales en servicio ${ }^{\star \star \star}$ del Hospital Universitario "Angel Larralde" hacia la carrera de Enfermería.

\section{Objetivo}

Determinar las actitudes status social, status económico, vocación y desarrollo científico de los bachilleres asistenciales en servicio hacia la carrera de Enfermería.

\footnotetext{
*Enfermera Jefe I (supervisora) del área de Pediatría Hospital Central Valencia "Dr. Enrique Tejera". Docente Contratada. Universidad de Carabobo. Facultad de Ciencias de la Salud. Escuela de Medicina, Valencia, Venezuela.

**Enfermera Il coordinadora del área de Enfermería del Hospital "Dr. Rafael Calles Sierra” - Coro - Falcon - Venezuela.

***Todo aquel egresado de educación media con el título de bachiller asistencial mención Enfermería, que ejerce funciones como profesional de esta carrera.
} 


\section{Metodología}

\section{Universo:}

El universo de la presente investigación fueron ciento treinta y ocho (138) bachilleres asistenciales en servicio, titulares de cargo del Hospital Universitario "Angel Larralde".

\section{Muestra:}

La selección de la muestra se realizó al azar simple, tomándose el 30\% del total del universo representado por cuarenta y un (41) bachilleres asistenciales.

\section{Método:}

El método empleado fue el descriptivo, por tratarse de un problema socio-educativo, que requiere de una explicación con base científico.

\section{Instrumento:}

En base al análisis y observaciones realizadas al instrumento de la prueba piloto, se elaboró el instrumento definitivo, el cual contiene:

Veinte cuatro (24) proposiciones.

La escala utilizada para la medición de las actitudes fue la escala de Lickert o método sigma de puntuación contentiva de cinco (5) alternativas: apruebo totalmente, apruebo en ciertos aspectos, indeciso, desapruebo en ciertos aspectos, desapruebo totalmente.

\section{Técnicas Estadísticas:}

Para la presentación y análisis de los datos se utilizó la técnica de proporción o porcentaje. Se tomaron como datos de significación estadística, aquellos resultados por encima del cuarenta por ciento (40\%), ubicados en las diferentes opciones de la escala de Lickert.

\section{Resultados:}

En la determinación de la actitud status social, más del ochenta por ciento $(82,8 \%)$ de los elementos encuestados estuvieron totalmente de acuerdo con la proposición "La ley del ejercicio profesional es indispensable para elevar el status socio-económico y académico de enfermería".

Las profesiones para elevar su status socio-económico requieren de un apoyo a través de la ley del ejercicio profesional, el cual enfermería aún no posee y éstos resultados evidencian que los bachilleres asistenciales reconocen esta necesidad.

Más de las tres cuartas partes (80\%) aprobó totalmente la proposición "socialmente vale más una buena enfermera que un mal médico", se evidencia el contenido afectivo de las actitudes, por lo que podemos inferir, que existe un alta auto-estima profesional en los elementos de la muestra.

Cerca de las tres cuartas $(74,2 \%)$ de la muestra. Estuvo totalmente en desacuerdo con la proposición "Enfermería avanzaría técnica y científicamente más rápido si la mayoría de sus egresados fuesen hombres". Estos resultados niegan el argumento teórico que sustenta que la mujer es relegada a ocupar un lugar secundario en las funciones productivas.

Más del cincuenta por ciento $(54,2 \%)$ de los encuestados aprobó totalmente las proposiciones "La enfermería es una carrera que tiene muchas posibilidades futuras de desarrollo y crecimiento socio-económico, científico y tecnológico" y "ser licenciado en Enfermería ofrece más prestigio profesional que ser bachiller asistencial".

La apertura reciente de la carrera de Enfermería en institutos públicos y privados de educación superior, ofrecen la posibilidad futura de avances científicos y técnicos y en consecuencia elevar el status socio-económico de Enfermería.

Por otra parte elevan el nivel educativo de enfermería a nivel superior implica elevar su prestigio profesional.

Estos datos refuerzan los resultados obtenidos por Salazar y Guerrero de Z. en 
su investigación, "Actitudes de los estudiantes de un liceo diversificado de Valencia hacia la profesión de enfermería" en el cual "La enfermería es una profesión nueva con grandes posibilidades de éxito para el futuro; fue la opinión en casi las tres cuartas partes $(73,71 \%)$ del total de la muestra". (1)

Cerca del cincuenta por ciento (48,5\%) de los elementos de la muestra estuvo parcialmente en que "La enfermería como carrera universitaria es conocida ampliamente". Tomando en cuenta que la Escuela de Enfermería de la Universidad de Carabobo inició sus actividades hace diecisiete (17) años, se justifica que los elementos de la muestra aprueben parcialmente la proposición.

De los elementos encuestados más del cuarenta por ciento (45.7\%) aprobó totalmente la proposición "El prestigio social de enfermería es menos que el de otras carreras universitarias".

El prestigio social de las profesiones esta determinado por el ingreso económico y enfermería recibe un salario inferior comparado con los demás profesionales del sector salud.

Se puede observar, en base a los análisis anteriores que las actitudes en el status social de bachilleres asistenciales en servicio del Hospital Universitario "Angel Larralde" hacia la carrera de enfermería es favorable de acuerdo a los porcentajes significativos obtenidos económico casi el noventa por ciento (88.5\%) desaprobó totalmente la proposición "Las enfermeras(os) son bien remunerados". Este resultado avala el planteamiento teórico de que enfermería es la profesión que menos remuneración recibe dentro del equipo de salud y resultados similares fueron obtenidos por Salazar y Guerrero Z., en un estudio hecho sobre "Las actitudes de los estudiantes de un liceo diversificado de Valencia hacia la profesión de enfermería"en donde "el bajo sueldo que devenga una enfermera es considerado por más de la mitad (60.57\%) de la investigada como uno de los motivos que incluyen en ellos para no escogencia de la carrera de enfermería" (2).

En cuanto a la proposición "EL egresado universitario de enfermería en el campo de trabajo recibe un sueldo igual al bachiller asistencial". Más del cincuenta por ciento (62.8\%) la desaprobó totalmente.

Esta última proposición fue control de la anterior. Estos resultados reflejan la situación actual de enfermería, el cual no discrimina su nivel académico en la asignación de sueldos.

Más del cuarenta por ciento (45.7\%) estuvo de acuerdo al aprobar totalmente la proposición "Estudiar enfermería en la Universidad trae muchos gastos". No existen argumentos teóricos que sustenten o nieguen estos resultados dada la existencia de investigaciones el tema.

Más del cuarenta por ciento (42.8\%) de los encuestados desaprobó totalmente la proposición "Las instituciones empleadoras ofrecen estímulos económicos por mejoramiento profesional". Estos datos refuerzan lo acotado anteriormente en las proposiciones acerca de las remuneraciones percibidas por los bachilleres asistenciales y el egresado universitario en enfermería.

Se observa en base a los análisis anteriores que las actitudes en el status económico de los elementos de la muestra hacia la carrera de enfermería, es desfavorable de acuerdo a los porcentajes significados obtenidos (ver tabla 2).

En la actitud vocacional casi el noventa por ciento (88.5\%) de la muestra aprobó totalmente la proposición "La vocación es decisiva en la escogencia de la carrera de enfermería; resultados similares obtuvieron Salazar y Guerrero de Z. en su investigación sobre "Las actitudes de los estudiantes de un liceo diversificado de Valencia hacia la profesión de enfermería" en donde "casi las tres cuartas partes $(72.11 \%)$ de la muestra seleccionada estuvieron de acuerdo con señalar la vocación como factor decisivo para la escogencia de la carrera de enfermería".

El sesenta por ciento (60\%) de los elementos de la muestra seleccionada, estuvieron totalmente de cuerdo con la proposición "si usted tuviese la oportunidad de estudiar una carrera universitaria estudiaría enfermería". Estos resultados reflejan el deseo de los bachilleres asistenciales en servicio de continuar su carrera a nivel superior.

En base al análisis de los resultados, la determinación de las actitudes en 
cuanto a la vocación, es favorable de cuerdo a los porcentajes significativos obtenidos (ver tabla 3).

Los elementos de la muestra en la actitud desarrollo científico, más del ochenta por ciento (82.8\%) estuvo totalmente de acuerdo en señalar que "La preparación científica de los enfermeros les permite proporcionar una mayor calidad de atención de los servicios" y casi cuartas partes (74.2\%) estuvo también totalmente de acuerdo con la proposición 'es necesaria la preparación universitaria para mejorar la calidad de atención de los servicios de enfermería".

Por ser la primera proposición control de la Segunda, los resultados evidencian la actitud favorable de los bachilleres ases asistenciales en servicio hacia la necesidad del desarrollo, a través de la preparación universitaria.

Casi las tres cuartas de la muestra seleccionada estuvo totalmente de acuerdo con la proposición "Frecuentemente trata usted de actualizarse asistiendo a cursos específicos de enfermería". Estos resultados revelan el interés de los elementos encuestados por su mejoramiento profesional.

Más de la mitad (51.4\%) de los elementos de la muestra estuvo totalmente de acuerdo en señalar que "para cumplir órdenes médicas no se requiere preparación universitaria". Estos resultados contradicen en análisis de los datos anteriores, por cuanto resulta incongruente que los elementos de la muestra reconozcan la necesidad y importancia de la preparación científica para mejorar la atención de los servicios de enfermería y por otra parte declaran que para cumplir la función independientemente de las órdenes médicas no se requiere preparación universitaria (ver tabla 4).

\section{Conclusiones y recomendaciones}

A través de la presente investigación se determinar algunas de las actitudes de los bachilleres asistenciales en servicios del Hospital Universitario "Angel Larralde" hacia la carrera de enfermería.

Los datos más resaltantes obtenidos para las actitudes: status social, status económico, vacación y desarrollo científico:

Status Social: más del ochenta por ciento $(82,8 \%)$ reconoce la necesidad que tiene la profesión de enfermería de una ley de ejercicio profesional que contribuya a elevar el status socioeconómico y académico de enfermería.

El contenido afectivo de las actitudes se evidenció a través de la alta auto-estima profesional de los elementos de la muestra al aprobar en un ochenta por ciento (80\%) la proposición "socialmente vale más una buena enfermera que un mal médico".

Cerca de las tres cuartas partes $(74,2 \%)$ niega que la condición femenina de enfermería sea un obstáculo para el técnico y científico de la profesión.

Más del cincuenta por ciento (54,2\%) afirma que el título de Licenciado en Enfermería es más prestigioso profesionalmente que el de bachiller asistencial. En esta proposición los elementos de la muestra reforzaron investigaciones anteriores que consideran que enfermería es una carrera con muchas posibilidades futuras de desarrollo y crecimiento socio-económico, científico y tecnológico.

Más del cuarenta por ciento $(45,7 \%)$ considera que el prestigio social de enfermería es menos que el de otras profesiones.

Status Económico: cerca del noventa por ciento (88,5\%) considera que las enfermeras(os) son mal remunerados, ratificando investigaciones anteriores donde un grupo de estudiantes de un Liceo diversificado de Valencia, señalaron que los bajos sueldos que reciben las enfermeras era uno de los motivos que influyen en ellos para la escogencia de la carrera.

Más del cincuenta por ciento $(62,8 \%)$ de los encuestados consideran la remuneración que recibe el bachiller asistencial y el licenciado en enfermería no esta de acuerdo con su preparación académica, ya que en el campo de trabajo ambos reciben la misma remuneración, dado que los organismos empleadores no discriminan el 
nivel académico en la asignación de sueldos.

Más del cuarenta por ciento $(42,8 \%)$ de los elementos de la muestra está de acuerdo con que las instituciones empleadoras no ofrecen estímulos por mejoramiento profesional.

Vocación: casi el noventa por ciento $(88,5 \%)$ consideran que la vacación es decisiva en la escogencia de la carrera de enfermería, semejantes resultados fueron obtenidos en investigaciones anteriores.

Se evidencia el deseo de los bachilleres asistenciales en servicio de continuar su carrera a nivel superior, ya que el sesenta por ciento (60\%) manifestó que de tener oportunidad de estudiar una carrera universitaria, estudiaría enfermería.

Desarrollo Científico: más del ochenta por ciento (82,8\%) afirmó que es necesario la preparación científica de las enfermeras para aumentar la calidad de la atención de los servicios y casi las tres cuartas partes $(74,2 \%)$ de los elementos de la muestra estuvo totalmente de acuerdo en que es necesaria la educación universitaria para mejorar la calidad de atención de los servicios de enfermería.

Más de las tres cuartas partes $(77,1 \%)$ trata frecuentemente de actualizarse asistiendo a cursos específicos de enfermería, estos resultados evidencian el interés de las enfermeras por su mejoramiento profesional.

\section{Recomendaciones}

Repetir el estudio con bachilleres adscritos al mayor empleador de enfermería, el M.S.A.S., dado que las condiciones de trabajo y los beneficios económicos del Ministerio de Sanidad y el I.V.S.S., son diferentes; y esto podría ser un factor determinante en las actitudes de los bachilleres asistenciales hacia la carrera de enfermería.

The purpose of this investigation was to study the attitudes of baccalaureate assistants concerning a Nursing career. The central objective was to determine the attitudes concerning social, economic and vocational status, and scientific development in Nursing. A total of 138 (30\%) baccalaureate, chosen at random, from the University Hospital "Angel Larralde" in Valencia, Venezuela, participated in this study. A descriptive plan was. The instrument was original and selected the School of Lickert. The statistical technique used was analysis of proportions. Results indicated favorable attitudes concerning social and vocational status and scientific development and unfavorable attitudes concerning economic status, according to the significant percentages obtained. This research was carried out as a requirement for graduation from the School of Nursing of the University of Carabobo in June 1990. 


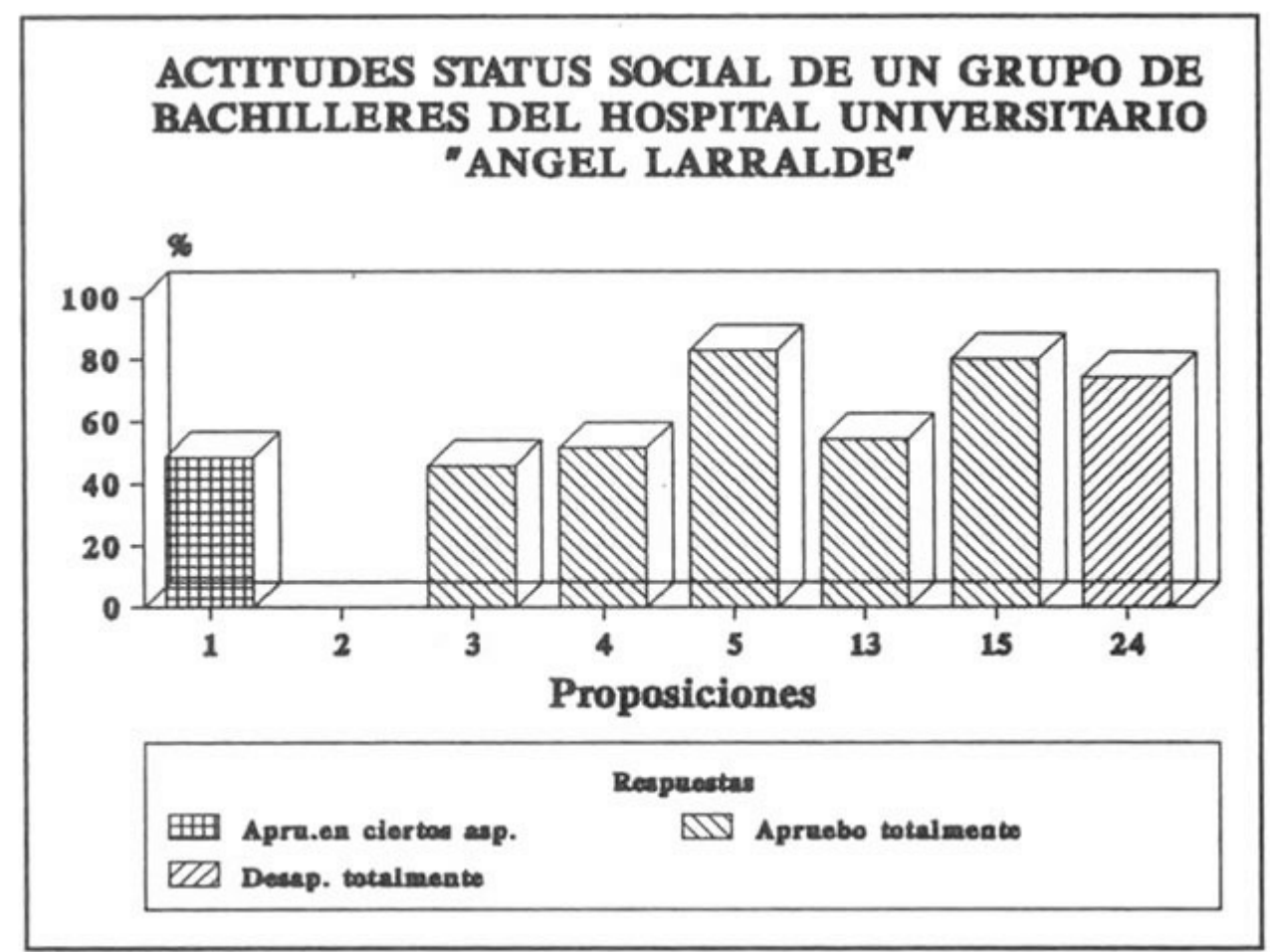

Tabla 1

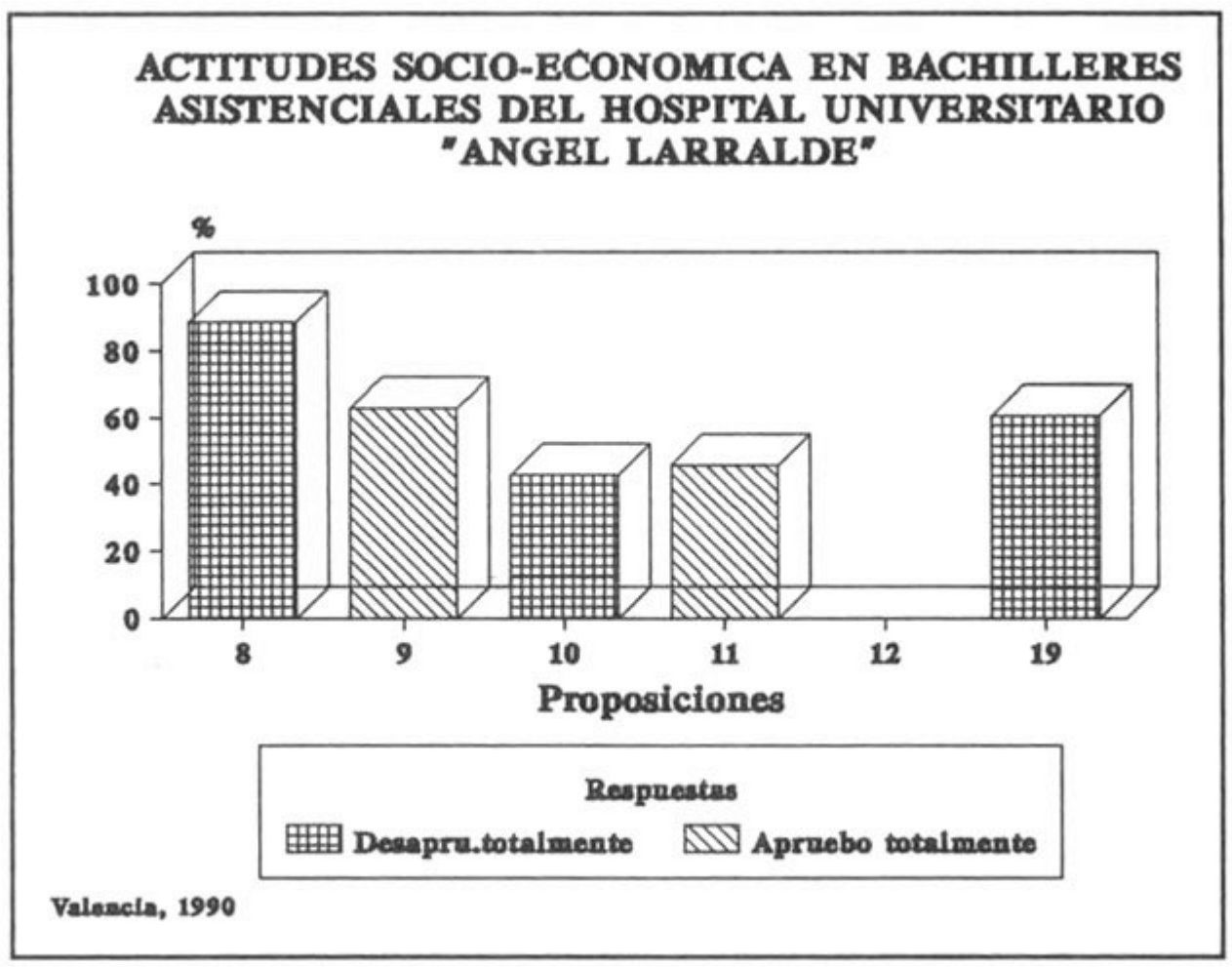

Tabla 2 


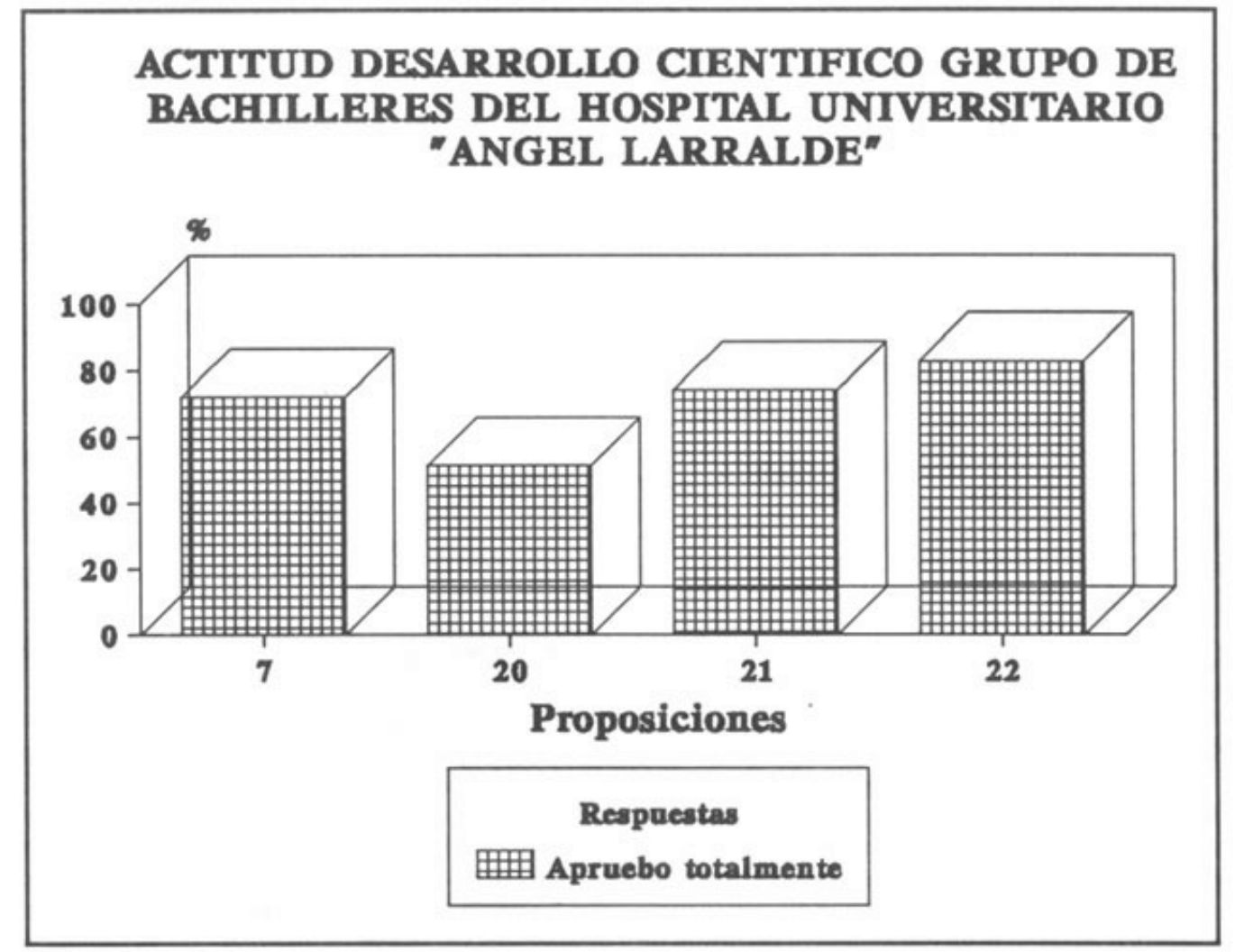

Tabla 3

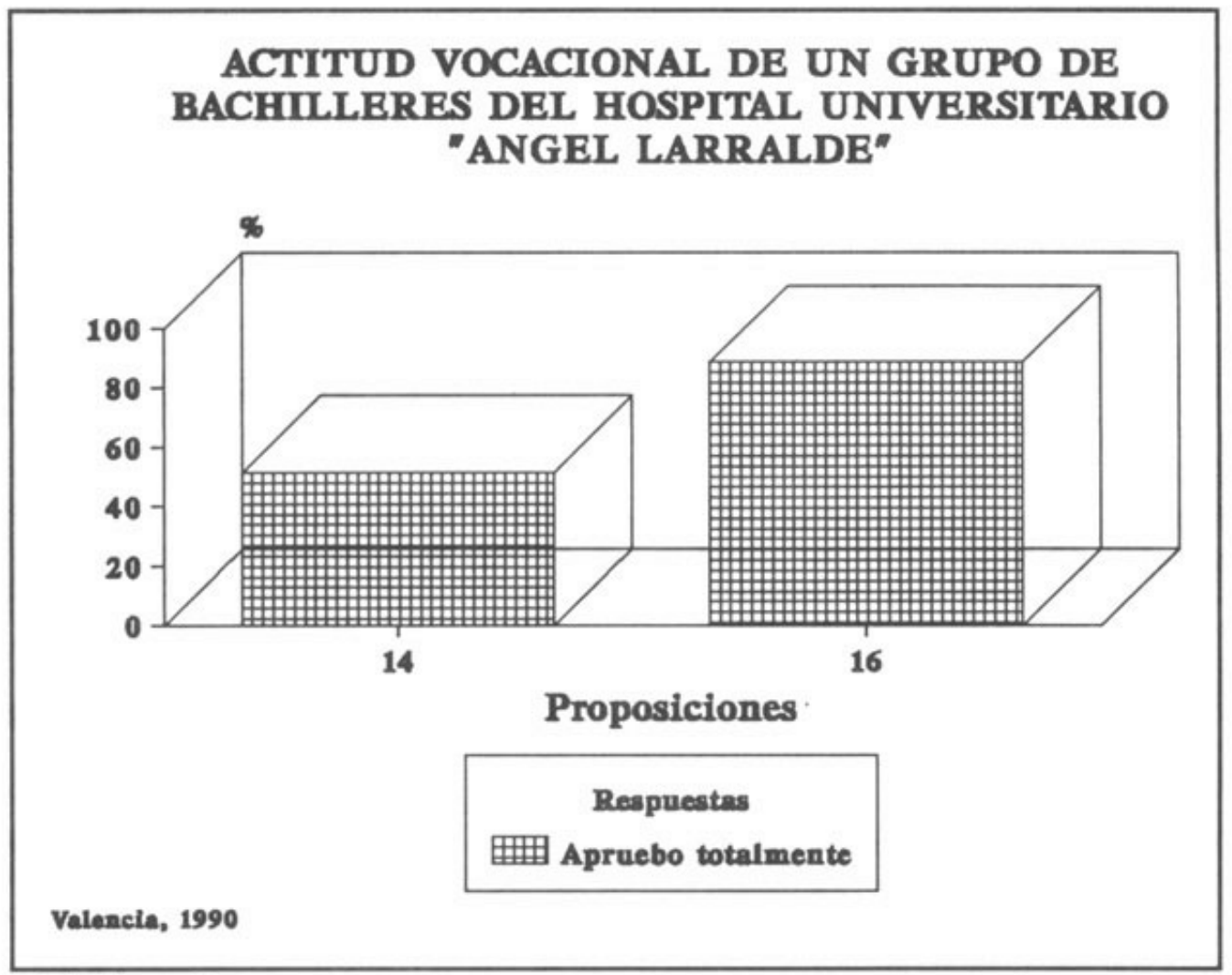

Tabla 4 
ANEXO I

PERSONAL DE ENFERMERIA DE VENEZUELA
ANO 1986

Aux. enfermeria
$67 \%$

Lic. on onfermeria $2 \%$

Bgressdos antes 1972

9\%

Bech. asistemciales

22\% 